\title{
Highly Enantioselective Adsorption of Small Prochiral Molecules on a Chiral Intermetallic Compound**
}

\author{
Jan Prinz, Oliver Gröning, Harald Brune, and Roland Widmer*
}

\begin{abstract}
Intrinsically chiral surfaces of intermetallic compounds are shown to be novel materials for enantioselective processes. Their advantage is the significantly higher thermal and chemical stability, and therefore their extended application range for catalyzed chiral reactions compared to surfaces templated with chiral molecular modifiers or auxiliaries. On the $P d_{1}$-terminated $P d G a(111)$ surface, room-temperature adsorption of a small prochiral molecule (9-ethynylphenanthrene) leads to exceptionally high enantiomeric excess ratios of up to $98 \%$. Our findings highlight the great potential of intrinsically chiral intermetallic compounds for the development of novel, enantioselective catalysts that can be operated at high temperatures and potentially also in harsh chemical environments.
\end{abstract}

Pronounced enantiodependence is a ubiquitous feature in biochemical processes and therefore a cardinal signature of life, where the question regarding the origin of the homochirality in biology still remains an unanswered scientific challenge of most profound implications. ${ }^{[1,2]}$ Among various scenarios it has been suggested that enantioselective adsorption on chiral crystal surfaces (for example, triclinic pyrite) in the prebiotic environment could have played a crucial role in this respect. ${ }^{[3]}$

From the technological perspective, the synthesis of enantiopure products is pivotal in the production of many pharmaceuticals, artificial flavors, agrochemicals, and materials for nonlinear optical devices. Chiral synthesis implies reactions that are asymmetric with regard to stereoisomerism $^{[4,5]}$ and therefore requires chiral template surfaces to achieve enantioselective heterogeneous catalysis. To date, such surfaces are commonly obtained by imparting chirality to non-chiral metal surfaces by the adsorption of chiral molecular modifiers or auxiliaries. ${ }^{[6,7]}$

[*] Dr. J. Prinz, Dr. O. Gröning, Dr. R. Widmer

Empa. Swiss Federal Laboratories for Materials Science and

Technology

Überlandstrasse 129, 8600 Dübendorf (Switzerland)

E-mail: roland.widmer@empa.ch

Homepage: http://www.surfaces.ch

Prof. Dr. H. Brune

Institute of Condensed Matter Physics, EPFL, Station 3

1015 Lausanne (Switzerland)

[***] We gratefully acknowledge inspiring discussions with Prof. KarlHeinz Ernst, theoretical simulations by Carlo Pignedoli, Aliaksandr Yakutovich, and Daniele Passerone, and funding by the Swiss National Science Foundation under Contract 200021-129511.

Supporting information for this article is available on the WWW under http://dx.doi.org/10.1002/anie.201410107.
This induced chirality for stereochemical processes is mainly achieved by chiral surface modifications using: 1) selfassembled chiral molecular layers ${ }^{[2,8-11]} 2$ ) deposition and alteration of chiral inorganic thin films; ${ }^{[12-14]}$ and 3) specific, high-index vicinal surfaces having chiral kink sites at the step edges of atomic terraces. ${ }^{[15-17]}$ While in these approaches, the chirality is limited to the surface layers or adsorbate layers, minerals, such as quartz ${ }^{[18]}$ and cinnabar, ${ }^{[19]}$ exhibit bulk chirality and were successfully studied towards enantioselective reactions. In comparison to the surface-modified catalysts, ${ }^{[20]}$ these materials potentially exhibit a higher density of chiral binding sites that is preserved even after grinding of the material into powders to increase the specific surface area. ${ }^{[19]}$ Another advantage is the significantly higher thermal and chemical stability, and therefore their extended application range for catalyzed chiral reactions. ${ }^{[18,21]}$ Some intermetallic compounds also have chiral bulk crystal structures. However, their potential in enabling stereochemical processes and therefore to possibly serve as novel, enantioselective heterogeneous catalysts has not been explored to this date. A highly interesting material in this regard is palladium-gallium (1:1), PdGa, which has recently attracted considerable interest owing to its high activity, selectivity, and stability in the catalytic semi-hydrogenation of ethyne, being an important step in the polyethylene production. ${ }^{[22]}$ Owing to its noncentrosymmetric bulk structure of the space group $P 2_{1} 3$, $\mathrm{PdGa}$ exists in two enantiomeric forms $\mathrm{A}$ and $\mathrm{B}$, which are denoted as PdGa:A and PdGa:B, respectively. ${ }^{[23-25]}$

Single crystals grown by the Czochralski method are available in $\mathrm{cm}^{3}$-sized monoliths ${ }^{[26]}$ and various low-index surfaces can be prepared to show large atomic terraces by standard ultrahigh vacuum (UHV) preparation using sputtering and annealing cycles. ${ }^{[24,25]}$ As no significant surfaces reconstructions occur, ${ }^{[24,25]}$ the bulk chirality is maintained at all of these surfaces, thus each surface unit cell exhibits a chiral atomic arrangement, as shown in Figure 1. This means that a high density of chiral binding centers is present on the bare surfaces, potentially leading to higher enantioselective catalytic activity compared to approaches based on the handedness of terrace step kink sites. ${ }^{[15-17]}$

$\mathrm{PdGa}: \mathrm{A}(111)$ is terminated by an atomic layer containing one isolated $\mathrm{Pd}$ atom per surface unit cell ${ }^{[24]}$ and accordingly this termination is denoted as $\mathrm{Pd}_{1}$ (Figure 1). These $\mathrm{Pd}$ atoms form a hexagonal lattice with a lattice constant of $0.69 \mathrm{~nm}$ and therefore the topmost layer of this surface is achiral. The structure of the 2nd and 3rd outermost surface layers, consisting of $\mathrm{Ga}$ and $\mathrm{Pd}$ trimers respectively, induces the surface chirality, which is indicated by red and cyan triangles in Figure 1. The complementary enantiomer of the PdGa:A(111) (abbreviated $\mathrm{A}: \mathrm{Pd}_{1}$ ) is the $\mathrm{PdGa}: \mathrm{B}(\overline{\mathrm{1}} \overline{1} \overline{1})$ surface 

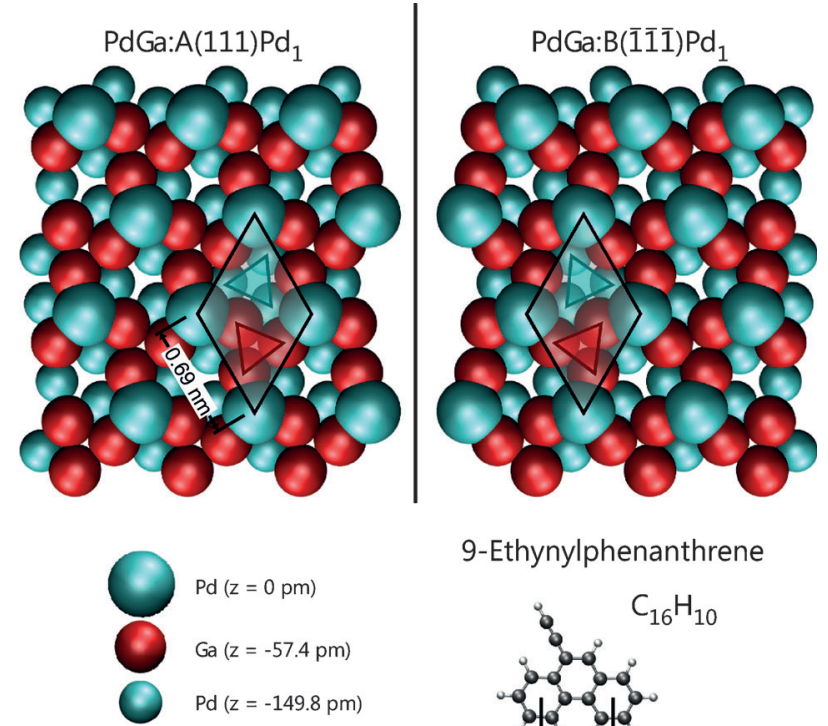

9-Ethynylphenanthrene

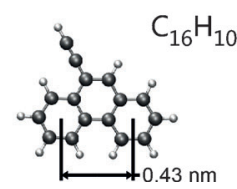

Figure 1. Surface structure of $\mathrm{PdGa}: \mathrm{A}(111) \mathrm{Pd}_{1}$ and its enantiomer $B(\overline{1} \overline{1}) \mathrm{Pd}_{1}$. The chiral arrangement within the hexagonal surface unit cell (black diamond) is highlighted by the cyan and red triangle, representing the Pd and $\mathrm{Ga}$ trimers of the subsurface layers, respectively. Upon adsorption on a surface, the prochiral molecule 9ethynylphenanthrene forms a chiral adsorption complex.

$\left(\mathrm{B}: \mathrm{Pd}_{1}\right)$. The relatively large size of the surface unit cell and the low density of the topmost Pd layer ensure that the chirality expressed in the deeper layers can still influence the molecular adsorption and reaction processes.

The effect of the intrinsic chirality of the $\mathrm{A}: \mathrm{Pd}_{1}$ and $\mathrm{B}: \mathrm{Pd}_{1}$ surfaces on enantioselective adsorption is investigated by deposition of the prochiral molecule 9-ethynylphenanthrene (9-EP; Figure 1). Prochirality is a property of planar molecules that possess in-plane but not out-of-plane mirror symmetry and are thus achiral. However, when deposited on a surface, the molecule forms a chiral adsorbate complex by lying either "face-up" or "face-down", forming either surface-enantiomer $R$ or $S$ as defined in an earlier study by Bebensee et al. ${ }^{[27]}$ For achiral substrates, energetically equivalent adsorption sites for the $R$ and $S$ surface-enantiomers are available, and thus they appear in equal abundance. ${ }^{[28]}$ To achieve an excess of one of the two enantiomeric forms, that is, enantioselectivity, the substrate must exhibit chiral binding sites that promote the preferred adsorption into either $R$ or $S$ configuration.

In the present case, the prochiral 9-EP molecule exhibits two distinct moieties, an aromatic ring system, and an ethynyl end group, which allows for different binding mechanisms to the substrate. Previous studies on ethyne $\left(\mathrm{C}_{2} \mathrm{H}_{2}\right)$ adsorption on the $\mathrm{Pd}_{1}$ surface revealed the preferred binding of this unsaturated hydrocarbon on-top of the outermost $\mathrm{Pd}$ atoms. ${ }^{[24]}$ In the case of $9-\mathrm{EP}$ on $\mathrm{Pd}_{1}$, the expectation is that the ethynyl group acts as anchor point to the outermost $\mathrm{Pd}$ atoms, while the aromatic ring system, because of its planar adsorption and spatial extent, acts as a probe for the surface chirality. As a consequence, the different surface chirality of $\mathrm{A}: \mathrm{Pd}_{1}$ and $\mathrm{B}: \mathrm{Pd}_{1}$, should each lead to an excess of one of the two surface enantiomers, $R$ or $S$, respectively. By employing high-resolution low-temperature scanning tunneling microscopy (STM), we demonstrate enantioselectivity by determining the handedness for a large number of single molecular adsorbates for different adsorption and sample annealing conditions.

Figure 2 shows high-resolution STM images of 9-EP deposited at room temperature (RT) with sub-monolayer coverage, imaged at $5 \mathrm{~K}$ on $\mathrm{A}: \mathrm{Pd}_{1}$ and $\mathrm{B}: \mathrm{Pd}_{1}$, respectively.
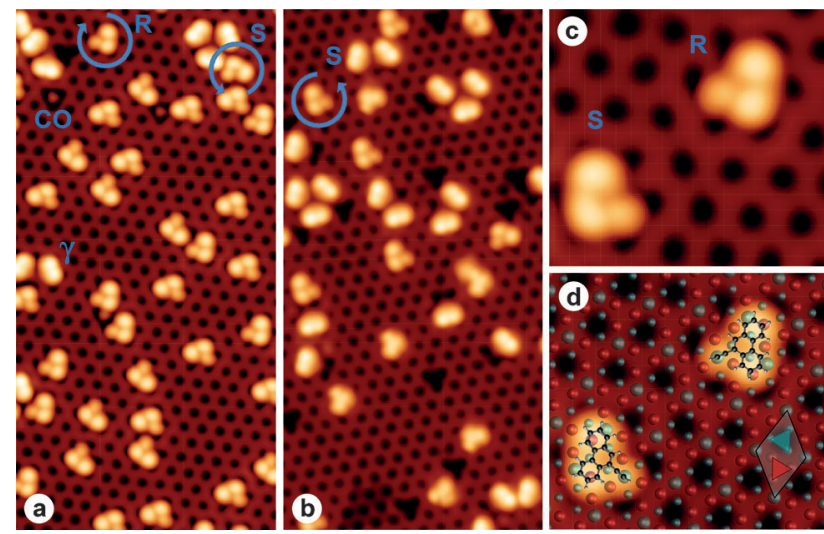

Figure 2. STM images $(10 \times 20 \mathrm{~nm}, 0.02 \mathrm{~V}, 2 \mathrm{nA}, T=5 \mathrm{~K})$ of 9 -EP adsorbed at room temperature on a) the $\mathrm{PdGa}: \mathrm{A}(111) \mathrm{Pd}_{1}$ surface and b) the $\mathrm{PdGa}: \mathrm{B}(\overline{\overline{1}} \overline{1} \overline{1}) \mathrm{Pd}_{1}$ surface. The different adsorbates are identified as clockwise $(R)$ and counterclockwise $(S)$ adsorption conformation, vacancy pinned molecules $(\gamma)$, and CO molecules. Some unoccupied vacancy defects (dark features) are visible in B. c) Zoom $(3.8 \times 3.5 \mathrm{~nm})$ showing $R$ and $S$ surface-enantiomers on $\left.A: P d_{1} . d\right)$ The structure overlay allows the adsorption position within the surface unit cell to be identified (cf. Figure 1).

Single 9-EP molecules can easily be identified and can be categorized in two groups: 1) molecules decorating $\mathrm{Pd}$ vacancies $^{[29]}$ on the surface and 2) molecules adsorbed on the defect free clean surface. The latter shows an asymmetric, three-lobe morphology (Figure 2c), which can be readily attributed to the $R$ and $S$ surface enantiomer of the 9-EP (Figure 2d). The former (denoted as $\gamma$ and indicated in Figure 2a) presents an elongated, two lobe structure from which the handedness of the molecule cannot be unambiguously determined. One, two, or three 9-EP molecules of this group can decorate a single Pd vacancy. As the vacancy presents a pronounced chiral atomic configuration and all of the $\gamma$-type molecules show the same STM morphology rotated by $120^{\circ}$, it is quite possible that they are predominantly of the same enantiomeric form, which however cannot be clearly identified by STM. Consequently our discussion regarding the enantioselectivity will concentrate on the $R$ and $S$ type molecules present on the defect free surface.

The handedness of these types can be inferred from the sense of rotation going from the largest to the smallest lobe, ${ }^{[27]}$ where the clockwise rotation from the large, over medium, to small protrusion is defined as $R$, and the counterclockwise sequence as $S$ surface enantiomer, as labeled in Figure $2 \mathrm{a}$ and b. For adsorption on $\mathrm{A}: \mathrm{Pd}_{1}$, shown in Figure $2 \mathrm{a}$, the excess of the $R$ surface enantiomer is evident $(R: S=215: 7$, for the full 
STM image), while $S$ is predominant on $\mathrm{B}: \mathrm{Pd}_{1}$ (Figure $2 \mathrm{~b}$; $R: S=2: 196)$. Owing to the threefold symmetry of the substrate, each surface enantiomer appears with equal probability in three orientations, which are rotated by $120^{\circ}$ to each other. Alternatively, $60^{\circ}$-rotated configurations with the centered phenanthrene ring atop a Ga trimer of the 2nd layer are observed. As the clockwise or anticlockwise rotations of these alternative adsorbates can still be identified, they are considered a subgroup of the $R$ and $S$ surface enantiomers in this study (see the Supporting Information for more details). For deposition at RT, the statistical analysis of molecules in $R$ and $S$ configuration yields an enantiomeric excess $e e(e e=|R-S| /(R+S))$ of 0.94 and 0.98 on $\mathrm{A}: \mathrm{Pd}_{1}$ and $\mathrm{B}: \mathrm{Pd}_{1}$, respectively.

Figure $2 \mathrm{c}$ shows a zoom of two surface-enantiomers. For the determination of the adsorption sites the surface atomic structure has been superimposed in Figure $2 \mathrm{~d}$. Its in-plane position and orientation has been identified by using adsorbed $\mathrm{CO}$ molecules as markers for the topmost Pd atoms $^{[30]}$ and by determination of the crystallographic structure of the subsequent atomic planes using LEED-I(V) (lowenergy electron diffraction). ${ }^{[2]}$ The combined information allows to unambiguously identify the topographic depression in the STM topography as the 3rd layer Pd trimer sites. For both surface-enantiomers, the ethynyl group is found atop the outermost Pd surface atoms, and the center phenanthrene ring atop a Pd trimer of the 3rd topmost atomic layer. Owing to the surface chirality, the position of the two outer phenyl rings with respect to the underlying substrate atoms is slightly different for the $R$ and $S$ surface enantiomers, respectively. The resulting adsorption energy difference seems to be sufficient for driving the observed and very pronounced enantioselectivity.

As the adsorption is carried out with the substrate at RT, but the determination of the enantiomeric excess is obtained from STM images measured at $5 \mathrm{~K}$ the question arises: At which temperature does the excess of the preferred surface enantiomer develop? To elucidate this question, temperature-dependent adsorption and annealing experiments were carried out. We deposited 9-EP on the A:Pd ${ }_{1}$ sample at $T=$ $135 \mathrm{~K}$ to investigate whether enantioselectivity is already achieved at the reduced available thermal energy of the substrate. The corresponding STM image in Figure 3 a reveals the presence of a large variety of adsorption conformations, with a comparable amount of $R$ and $S$ surface- enantiomers, meaning that no significant enantioselectivity is observed. Thus, the reaction mechanism is, at least partly, driven by the temperature of the substrate and the molecules shown in Figure $3 \mathrm{a}$ are frozen in a metastable adsorption state. Postannealing of the sample prepared at low temperatures to $300 \mathrm{~K}$ for $80 \mathrm{~min}$ leads to an increase of the excess of $R$ over $S$ and $e e=0.77$. However, the very high enantioselectivity that is observed for room-temperature deposition (Figure $2 \mathrm{a}$ and b) is not reproduced. Therefore, even very long post-annealing is not as efficient as adsorption directly at the annealing temperature. This indicates that the adsorption process plays a role in overcoming the barrier for transformation to the energetically favored surface enantiomer of the adsorbate complex on the respective surface.

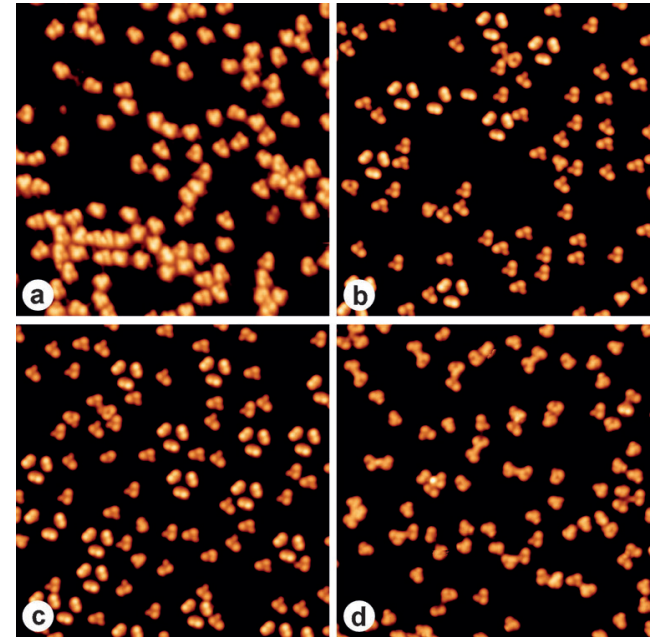

Figure 3. STM images $(T=5 \mathrm{~K}, 20 \times 20 \mathrm{~nm}, 0.2 \mathrm{~V}, 2 \mathrm{nA})$ of 9-EP after a) deposition at $135 \mathrm{~K}$ and b) at $300 \mathrm{~K} . \mathrm{c}$ ), d) The same sample as in $\mathrm{B}$ after post-annealing for $10 \mathrm{~min}$ to $370 \mathrm{~K}$ and $490 \mathrm{~K}$, respectively.

Higher annealing temperatures were tested to check whether enantioselectivity is maintained or whether new molecular species are formed on this catalytically active surface. To this end, we performed stepwise sequential postannealing of the sample that showed high enantioselectivity after room temperature deposition (Figure $3 \mathrm{~b}$ ). Figure $3 \mathrm{c}$ shows the sample after $10 \mathrm{~min}$ post-annealing to $370 \mathrm{~K}$, resulting in a slightly reduced enantioselectivity $(e e=0.83)$ as compared to sample after RT deposition. After further annealing to $490 \mathrm{~K}$ (Figure $3 \mathrm{~d}$ ), a large amount of the adsorbed molecules show a changed STM appearance, which is possibly due to dehydrogenation, and does not allow for unambiguous identification of the handedness. Furthermore, the formation of molecular dimers is observed, built from $R$ and $S$ surface-enantiomers in racemic ratios, with the molecules connected through their ethynyl groups. For $T>490 \mathrm{~K}$, almost all adsorbates have formed dimers and larger hydrocarbon agglomerates, while the overall amount of adsorbed carbon is maintained (see the Supporting Information). In all of the experiments, no significant decoration of step edges or kink sites was observed. STM experiments at room temperature (see the Supporting Information) revealed that only the molecules bound to surface vacancies $(\gamma)$ are not diffusing, while other adsorbates are mobile and thus cannot be individually resolved by STM.

To quantify the enantioselectivity of the $\mathrm{Pd}_{1}$ surface towards the adsorption of 9-EP, we evaluated and assigned a large number of adsorbates $(N \approx 400)$ for each of the experiments performed at different adsorption and annealing temperatures. In every case all of the molecules have been classified in the categories $R, S, \gamma$, and dimers. 9-EP molecules with an adsorbate configuration that cannot be unambiguously determined from their topographic appearance in STM are classified as unidentified. The result is shown as vertical colored bars in Figure 4, representing the normalized fraction of adsorbate configurations $R, S, \gamma$, and molecules that have formed dimers. At the top of the bars, the amount of unidentifiable adsorbates is given for each experiment. Based 


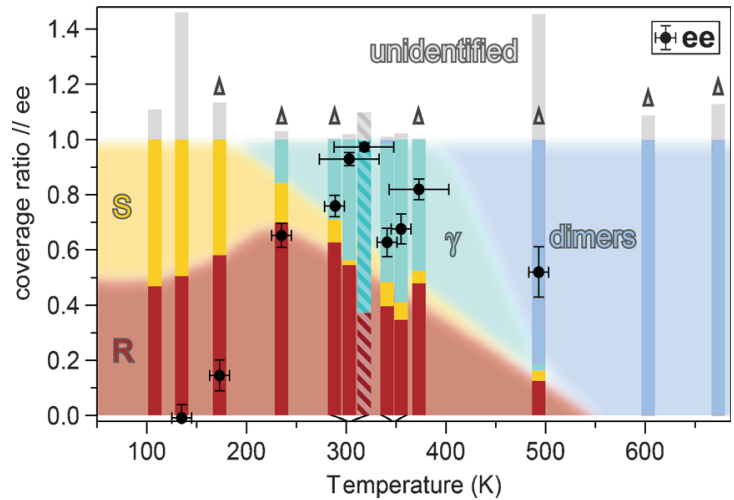

Figure 4. Normalized abundances for the $R$ and $S$ surface enantiomers, as well as molecules pinned to surface vacancies $(\gamma)$ and dimers. Annealed samples are marked by triangles. A phase diagram, based on the data of the different adsorbate configuration ratios, is presented as background. All bars represent the $A: \mathrm{Pd}_{1}$ surface apart from the striped bar representing $\mathrm{B}: \mathrm{Pd}_{1}$ (colors for $R$ and $S$ are inverted for clarity). The enantiomeric excess $(e e)$ is given for each experiment as black circles. Error propagation of the counting errors in the number of $R$ and $S$ surface enantiomers was used to compute the error for $e e$.

on the temperature-dependent quantities, a tentative phase diagram was constructed for the 9-EP species on $\mathrm{A}: \mathrm{Pd}_{1}$ (see the background in Figure 4). The ee value is evaluated from the observed amount of $R$ and $S$ surface enantiomers and shown for each experiment as black marker with statistically computed errors and estimated errors for temperature. The graph shows the transition from the racemic mixture of $R$ and $S$ surface enantiomers at low temperatures, over a region yielding high enantioselectivity situated around $300-350 \mathrm{~K}$, to the formation of dimers for $T>\approx 450 \mathrm{~K}$. The appearance of $\gamma$ type molecules, that is, molecules pinned at vacancies, at intermediate substrate temperature is linked to the increase in mobility. They are not taken into account in the evaluation of $e e$, and their role to the formation of the preferred surface enantiomer species remains unknown.

The data reveals that a temperature window of about $\pm 100 \mathrm{~K}$ around room temperature yields $e e>0.6$. For roomtemperature deposition, we find a high $e e$ of $0.98 \pm 0.01$ for $\mathrm{B}: \mathrm{Pd}_{1}$, while for the same experiment on the form A sample $e e=0.94 \pm 0.02$ is observed. The remarkably strong enantioselective adsorption can be compared to deposition of the same molecule on the achiral $\mathrm{Cu}(111)$ surface, where Bebensee et al. found equivalent quantities of $R$ and $S$ (see Ref. [27] and the Supporting Information therein).

Deposition of the molecules on the cold $\mathrm{A}: \mathrm{Pd}_{1}$ surface $(135 \mathrm{~K})$ and subsequent annealing to room temperature for $80 \mathrm{~min}$ results in $e e \approx 0.77 \pm 0.04$ (left bar for $300 \mathrm{~K}$ in Figure 4). Interestingly, the temperature onset, where enantioselectivity is observed, coincides with the occupation of surface vacancies $(\gamma)$, indicating that the mobility of molecules might play a role in the enantioselective transformation from $S$ to $R$. The reduction of $e e$ for annealing temperatures above $400 \mathrm{~K}$ can be explained by the entropic disorder of the equilibrium occupation of a two-level system with small energy difference ( $R$ and $S$ ). The fact that this reduction is still observed after cooling to $5 \mathrm{~K}$ with an estimated temperature ramp of about $1-5 \mathrm{~K} \mathrm{~s}^{-1}$, shows that the molecules were frozen in their position during cool-down, which indicates a low frequency for the $R$ to $S$ transformation mechanism.

Density functional theory calculations $\left(\mathrm{PBE}^{[31]}\right.$ with empirical van der Waals corrections ${ }^{[32]}$ ) have successfully identified the experimentally observed absorption configuration of the surface enantiomers. However, the calculated energy difference for the $R$ - to $S$-configuration of $15 \mathrm{meV}$ is too small to explain the observed selectivity. Further theoretical investigations are needed and ongoing to elucidate the adsorption energy landscape of this system.

In conclusion, we have demonstrated remarkably high enantioselective adsorption of a prochiral molecule on the bare chiral surface of an intermetallic compound at room temperature. The adsorption of 9-etynylphenanthrene on the A(111) surface and its mirror symmetric equivalent the $\mathrm{B}(\overline{1} \overline{1} \overline{1})$ surface of $\mathrm{PdGa}$ has shown enantiomeric excess of 0.94 and 0.98 for the $R$ and the $S$ surface enantiomers, respectively. By studying the temperature dependence of the process, we showed that the racemic molecule mixture formed by adsorption of the 9-EP at temperatures below $120 \mathrm{~K}$ is efficiently transformed to an almost enantiopure ensemble at room temperature.

This observation shows that the bulk chirality of PdGa is expressed sufficiently strong at its surfaces to yield highly enantioselective processes. In combination with the catalytic properties of intermetallic compounds in general and $\mathrm{PdGa}$ in particular, this opens the perspective of chiral heterogeneous catalysis (for example, surface-mediated enantioselective $\mathrm{C}=$ $O$ hydrogenation in $\alpha$ - or $\beta$-ketoacids ${ }^{[33-35]}$ or asymmetric hetero-Diels-Alder Coupling reactions ${ }^{[36-38]}$ ) under operation conditions beyond the ones allowed by catalyst surfaces based on functionalization with organic chiral modifiers.

Keywords: adsorption - chirality · enantioselectivity · intermetallic compounds · scanning probe microscopy

How to cite: Angew. Chem. Int. Ed. 2015, 54, 3902-3906 Angew. Chem. 2015, 127, 3974-3978

[1] D. G. Blackmond, Cold Spring Harbor Perspect. Biol. 2010, 2.

[2] I. Weissbuch, M. Lahav, Chem. Rev. 2011, 111, 3236-3267.

[3] G. Wächtershäuser, Prog. Biophys. Mol. Biol. 1992, 58, 85-201.

[4] B. D. Vineyard, W. S. Knowles, M. J. Sabacky, G. L. Bachman, D. J. Weinkauff, J. Am. Chem. Soc. 1977, 99, 5946-5952.

[5] R. Noyori, S. Hashiguchi, Acc. Chem. Res. 1997, 30, 97-102.

[6] T. Sugimura in Handbook of Asymmetric Heterogeneous Catalysis, Wiley-VCH, Weinheim, 2008, pp. 357-382.

[7] I. Weissbuch, L. Addadi, M. Lahav, L. Leiserowitz, Science 1991, $253,637-645$

[8] K. H. Ernst, Phys. Status Solidi B 2012, 249, 2057-2088.

[9] R. Raval, Chem. Soc. Rev. 2009, 38, 707-721.

[10] L. Pérez-García, D. B. Amabilino, Chem. Soc. Rev. 2007, 36, $941-967$.

[11] S. De Feyter, A. Gesquière, K. Wurst, D. B. Amabilino, J. Veciana, F. C. De Schryver, Angew. Chem. Int. Ed. 2001, 40, 3217-3220; Angew. Chem. 2001, 113, 3317-3320.

[12] S. K. Sarkar, N. Burla, E. W. Bohannan, J. A. Switzer, Electrochim. Acta 2008, 53, 6191-6195.

[13] J. A. Switzer, H. M. Kothari, P. Poizot, S. Nakanishi, E. W. Bohannan, Nature 2003, 425, 490-493. 


\section{Angewandte}

[14] R. Widmer, F. J. Haug, P. Ruffieux, O. Groning, M. Bielmann, P. Groning, R. Fasel, J. Am. Chem. Soc. 2006, 128, 14103-14108.

[15] S. J. Jenkins, S. J. Pratt, Surf. Sci. Rep. 2007, 62, 373-429.

[16] A. J. Gellman, Y. Huang, X. Feng, V. V. Pushkarev, B. Holsclaw, B. S. Mhatre, J. Am. Chem. Soc. 2013, 135, 19208-19214.

[17] A. Ahmadi, G. Attard, J. Feliu, A. Rodes, Langmuir 1999, 15, $2420-2424$.

[18] R. M. Hazen, D. S. Sholl, Nat. Mater. 2003, 2, 367-374.

[19] H. Shindo, Y. Shirota, K. Niki, T. Kawasaki, K. Suzuki, Y. Araki, A. Matsumoto, K. Soai, Angew. Chem. Int. Ed. 2013, 52, $9135-$ 9138; Angew. Chem. 2013, 125, $9305-9308$.

[20] A. Baiker, Catal. Today 2005, 100, 159-170.

[21] T. Mallat, E. Orglmeister, A. Baiker, Chem. Rev. 2007, 107, $4863-4890$.

[22] M. Armbrüster, K. Kovnir, M. Behrens, D. Teschner, Y. Grin, R. Schlögl, J. Am. Chem. Soc. 2010, 132, 14745-14747.

[23] M. Armbrüster, H. Borrmann, M. Wedel, Y. Prots, R. Giedigkeit, P. Gille, Z. Kristallogr. New Cryst. Struct. 2010, 225, $617-$ 618.

[24] J. Prinz, C. A. Pignedoli, Q. S. Stoeckl, M. Armbrüster, H. Brune, O. Gröning, R. Widmer, D. Passerone, J. Am. Chem. Soc. 2014, 136, 11792-11798.

[25] D. Rosenthal, R. Widmer, R. Wagner, P. Gille, M. Armbrüster, Y. Grin, R. Schlögl, O. Gröning, Langmuir 2012, 28, 6848-6856.

[26] P. Gille, T. Ziemer, M. Schmidt, K. Kovnir, U. Burkhardt, M. Armbrüster, Intermetallics 2010, 18, 1663-1668.

[27] F. Bebensee, C. Bombis, S. R. Vadapoo, J. R. Cramer, F. Besenbacher, K. V. Gothelf, T. R. Linderoth, J. Am. Chem. Soc. 2013, 135, 2136-2139.
[28] W. Xu, R. E. A. Kelly, H. Gersen, E. Laegsgaard, I. Stensgaard, L. N. Kantorovich, F. Besenbacher, Small 2009, 5, 1952-1956.

[29] J. Prinz, R. Gaspari, C. A. Pignedoli, J. Vogt, P. Gille, M. Armbrüster, H. Brune, O. Gröning, D. Passerone, R. Widmer, Angew. Chem. Int. Ed. 2012, 51, 9339-9343; Angew. Chem. 2012, 124, 9473-9477.

[30] J. Prinz, R. Gaspari, Q. S. Stoeckl, P. Gille, M. Armbrüster, H. Brune, O. Gröning, C. A. Pignedoli, D. Passerone, R. Widmer, J. Phys. Chem. C 2014, 118, 12260-12265.

[31] J. P. Perdew, K. Burke, M. Ernzerhof, Phys. Rev. Lett. 1997, 78, $1396-1396$.

[32] S. Grimme, J. Antony, S. Ehrlich, H. Krieg, J. Chem. Phys. 2010, 132,154104

[33] G. Jannes, V. Dubois, Chiral reactions in heterogeneous catalysis, Plenum, New York, 1995.

[34] H. U. Blaser, Tetrahedron: Asymmetry 1991, 2, 843-866.

[35] H. U. Blaser, Handbook of Heterogeneous Catalysis, Vol. 5, Wiley-VCH, Weinheim, 1997.

[36] E. J. Corey, Angew. Chem. Int. Ed. 2002, 41, 1650-1667; Angew. Chem. 2002, 114, 1724-1741.

[37] K. C. Nicolaou, S. A. Snyder, T. Montagnon, G. Vassilikogiannakis, Angew. Chem. Int. Ed. 2002, 41, 1668-1698; Angew. Chem. 2002, 114, 1742-1773.

[38] H. M. I. Osborn, D. Coisson, Mini-Rev. Org. Chem. 2004, 1, 41 54.

Received: October 15, 2014

Revised: December 7, 2014

Published online: January 29, 2015 